\title{
Co-operative Amplify and Forward Relaying Strategy for Mobile Adhoc Network for Efficient Communication
}

\author{
Vivek K. Dethe ${ }^{1}$, Dr. C.V. Ghule ${ }^{2}$, Dr. Om Prakash ${ }^{3}$ \\ Research Scholar, ECE Department, Shri. JJT University, Jhunjhunu, Rajasthan, India ${ }^{1}$ \\ Research Guide, ECE Department, Shri. JJT University, Jhunjhunu, Rajasthan, India ${ }^{2,3}$
}

\begin{abstract}
For enhancing the BER performance of a wireless network by reducing the effects of fading, transmit diversity is an effective way [1]. Due to size, hardware and cost constraints, majority of the wireless devices (i.e. mobile handsets, etc) are limited to only one antenna. Co-operative communication can be used and incorporated to achieve the transmit diversity by utilizing single antenna wireless devices to share their antennas during session. Such an arrangement creates a virtual MIMO (multiple-input and multiple-output) system [2] [3]. In this paper, we will discuss about the cooperative communications and analyze the performance of amplify and forward protocol for single relay model in idealistic condition which gives way forward to tackle the challenges in Spectrum allocation, Improvement of channel capacity, Extension of range, energy efficient Communications etc.
\end{abstract}

Keywords: Co-operative Communication, Multiple Input Multiple Output Systems, Transmit Diversity, Relay, Amplify and Forward, Adhoc Networks etc.

\section{INTRODUCTION}

"The increasing demand for wireless Internet services, adapting upon a limited feedback from the destination along with rapid growth of communications and gadgets, terminal are summarized in the paper [7]. Performance are creating opportunities for innovative research efforts characterizations in terms of outage events and associated on the design of novel wireless communication systems outage are developed [1], so that strengths of the signals architectures for high speed, reliable and cost effective are demonstrated, focusing on the high signal-to-noise transmission solutions" [4]. The introduction and rapid ratio (SNR) regime. It was also shown that cooperative development of MIMO (multiple-input and multiple- diversity protocols efficient as compared to fixed MIMO output) systems has results in improvements in reliability devices. Therefore, using distributed antennas, significant and throughput for Ad-hoc networks. The Multiple benefits of space diversity without need for physical arrays antennas can be utilized both at the transmitter and the can be achieved, with a cost of additional receive receiver side to gain the diversity. However, this technique hardware.

is advantageous for cellular base stations, but not feasible presently for mobile devices, due to their sizes and power constraints [5]. The improved performance can be achieved by new technique known as multi-user cooperative diversity in which a single antenna of wireless devices are used to get the transmit diversity benefits by sharing their physical resources through a virtual transmit and receive antenna array. The major benefit of such technique includes the diversity - because different paths practically fades independently, beam forming gain and interference mitigation [1] [5].

According to the paper [6], authors consider lowcomplexity cooperative protocols that reduce induced fading by multipath propagation in wireless networks. The basic idea of the technique is that the signals from transmitter and receivers can be relayed with cooperation amongst them. A few fixed relaying methodologies, such as amplify-and-forward, decode-and-forward, selection relaying schemes along with incremental relaying schemes

\section{CO-OPERATIVE COMMUNICATION}

The history of the co-operative communication can found its deep roots to the groundbreaking work of Van der Meulen [6], introduced the concept of relay channel, which utilize relay between a source and destination to facilitate the information transfer from source to destination. Later, Cover and El Gamal [7] investigated the relay channel model, and proposed a fundamental relaying strategies such as "Amplify and Forward (AF), Decode and Forward (DF) and Compress and Forward (CF)".

In conventional communication, data exchange takes place between the source and destination, and assistance to one another is not provided as shown below (Figure 1).

However, practically number of wireless neighboring nodes exists in a wireless communication network, which could be of great utilization towards cooperative communication. 
ISO 3297:2007 Certified

Vol. 5, Issue 2, February 2017

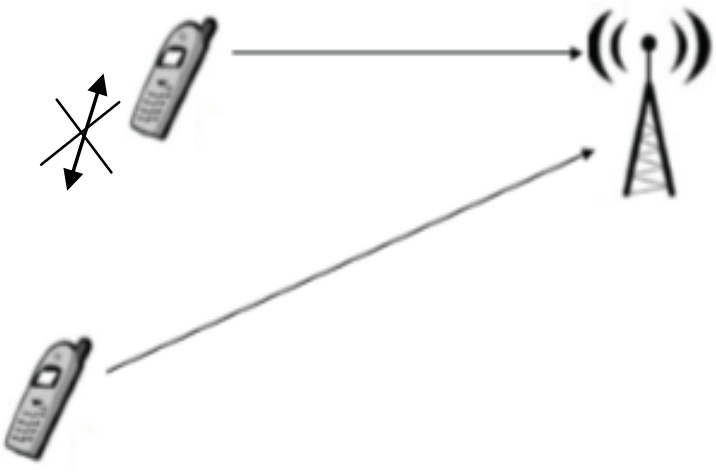

Figure 1. Conventional Communication

When one node transmits, all the nearby nodes heard it.Here Cooperative Communication targets to process and forward the received information to the respective destination and creates the diversity in space called as spatial diversity and results inenhancement of performance.

The concept of the cooperative communication is suggested in Figure 2. [4]

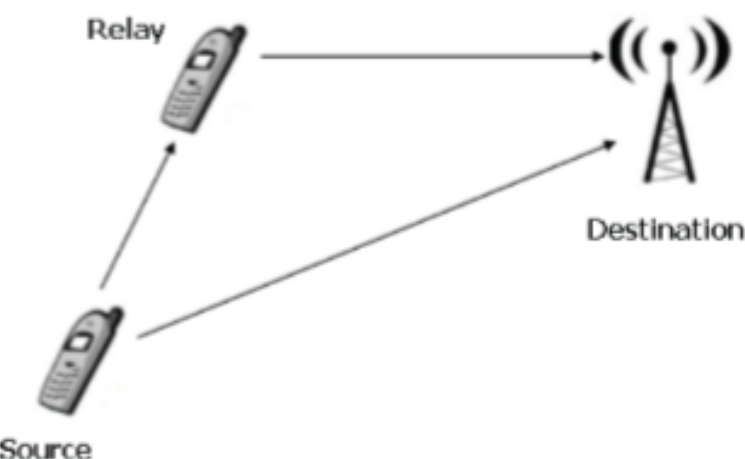

Figure 2. Cooperative Communication

As depicted in Figure 2, the source ' $\mathrm{S}$ ' is transmitting data to the destination ' $\mathrm{D}$ ', while the relay station (another mobile user) ' $R$ ' is also Receiving the transmitted signal from Source.

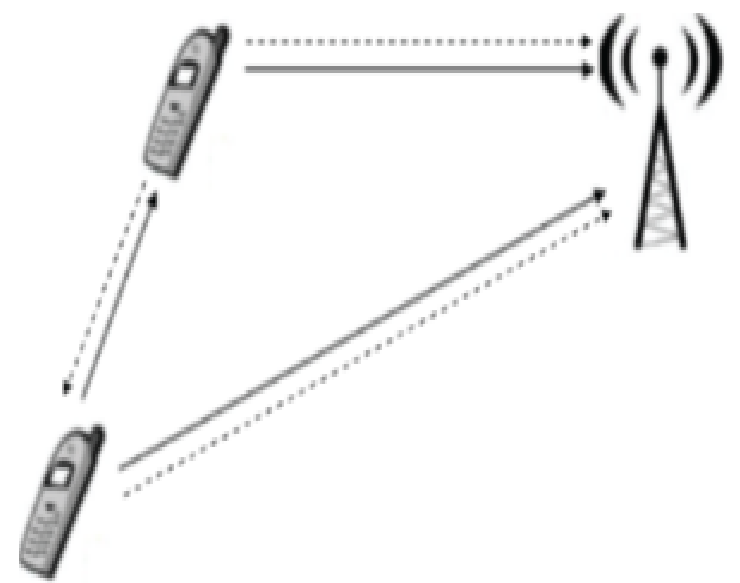

Figure3. Cooperative Communication, where each mobile act both as a user and relay.
At The relay station also process and forward this message to the destination, where both of the received signals are combined. As both copies of the signals are transmitted through independent paths, this results into spatial diversity. In cooperative communication, each wireless user is assumed to transmit its own data as well as act as a cooperative agent (relay) for the other user (Figure 3). [1] [8]

\section{COOPERATIVE TRANSMISSION PROTOCOLS}

Cooperative transmission protocols describes that how the received data is processed at the relay station, before forwarding it to the destination. In this article, we will review and analyse the performance of amplify and forward cooperative strategy in brief as compared to direct path.

TABLE I. Summary of outage probability approximations for statistically symmetric networks

\begin{tabular}{|l|c|c|}
\hline Protocol & $\left.\begin{array}{l}\text { Pout }^{\text {ounR }} \\
\text { highSNR }\end{array} \mathrm{SNorm}_{\text {Norm }}, \mathrm{R}\right)$, & $\begin{array}{l}\mathbf{P}^{\text {out }}\left(\mathrm{SNR}, \mathrm{R}_{\text {Norm }}\right), \\
\text { high } \mathrm{SNR}\end{array}$ \\
\hline Direct & $1 / \mathrm{SNR}_{\text {Norm }}$ & $1 / \mathrm{SNR}^{\left(1-\mathrm{R}_{\mathrm{Norm}}\right)}$ \\
\hline $\begin{array}{l}\text { Amplify- } \\
\text { and- } \\
\text { Forward }\end{array}$ & $\begin{array}{c}\left(2^{\mathrm{R}}+1\right)^{2} \\
/ \mathrm{SNR}_{\text {Norm }}^{2}\end{array}$ & $1 / \mathrm{SNR}^{2\left(1-2 \mathrm{R}_{\mathrm{Norm}}\right)}$ \\
\hline
\end{tabular}

\section{Amplify and Forward Strategy}

As the name suggests, in the Amplify and Forward technique simply amplify the signal received by the relay and then forward it to the destination. This technique was proposed by J. N. Laneman and G. W. Wornell [9], and is most suited for relays having minimal computing power. However, the noise in the signal is also amplified at the relay station in this arrangement which is major drawback, and the destination receives two independently faded versions of the signal.

More detailed discussion has been given by J. N. Laneman and G. W. Wornell [9] for a better understanding of the Amplify and Forward technique.

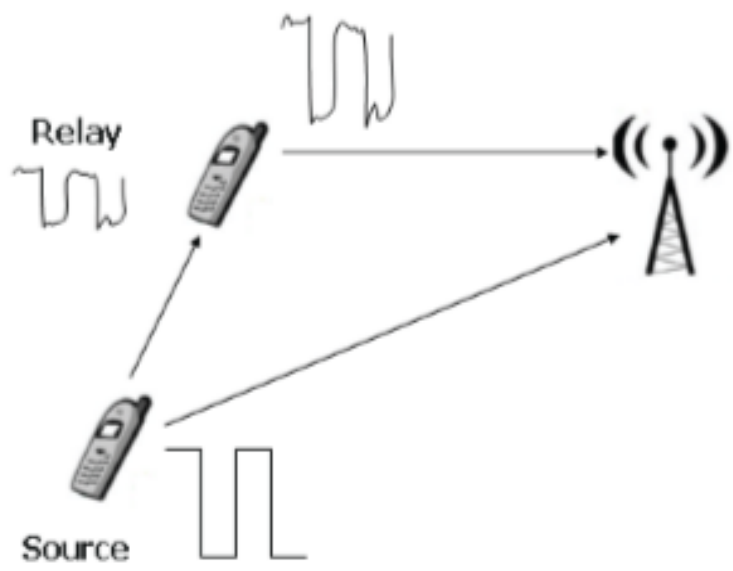

Figure 4. Amplify and Forward Technique. 


\section{METHODOLOGY}

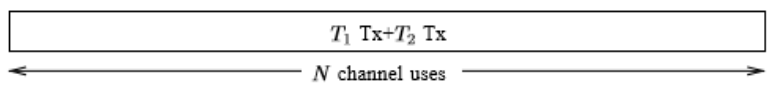

(a)

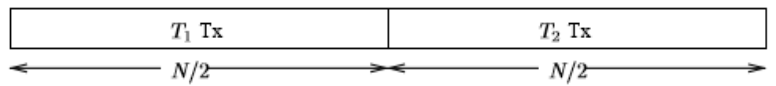

(b)

\begin{tabular}{|l|c|c|c|}
\hline$T_{1} \mathrm{Tx}+T_{2} \mathrm{Rx}$ & $T_{2}$ Relay & $T_{2} \mathrm{Tx}+T_{1} \mathrm{Rx}$ & $T_{1}$ Relay \\
\hline
\end{tabular}

(c)

Figure5. Example time-division channel allocations for (a) direct transmission with interference, (b) orthogonal direct transmission, and (c) orthogonal cooperative diversity

For direct transmission, the channel cab be modelled as:

$$
y_{d}[n]=a_{s, d} x_{s}[n]+z_{d}[n]
$$

for, say, $\mathrm{n}=1, \ldots, \mathrm{N} / 2$, where $x_{s}[n]$ represents the source transmitted signal, and $y_{d}[n]$ represents the destination received signal.

The another node transmits for $\mathrm{n}=\mathrm{N} / 2+1, \ldots, \mathrm{N}$ as Fig. 2(b) depicts. Thus, each node uses only half of the available resources of the channel.

For cooperative diversity, the channel model for first half of the block is as below:

$$
\begin{aligned}
& y_{r}[n]=a_{s, r} x_{s}[n]+z_{r}[n] \\
& y_{d}[n]=a_{s, d} x_{s}[n]+z_{d}[n]
\end{aligned}
$$

for, say, $\mathrm{n}=1, \ldots, \mathrm{N} / 4$, where $x_{s}[n]$ represents the source transmitted signal and $y_{r}[n]$ and $y_{d}[n]$ reprenets the relay and destination received signals, respectively.

For the second half of the block, the channel model of the received signal is:

$$
y_{d}[n]=a_{r, d} x_{r}[n]+z_{d}[n]
$$

for $\mathrm{n}=\mathrm{N} / 4+1, \ldots, \mathrm{N} / 2$, where $x_{r}[n]$ represents the relay transmitted signal and $y_{d}[n]$ is the destination received signal.

In second half of the block, the source and relays are reversed for their roles, as Fig. 2(c) depicts. Note here that, while again half the resources of channel are allocated to each source nodes for transmission to its destination, only a quarter of the resources are available for communication to its relay.

In (1)-(4), $a_{i, j}$ represents the effects of path-loss, shadowing, and frequency nonselective fading, and $z_{j}[n]$ represents the effects of receiver noise and other forms of interference in the system, where $i \in\{\mathrm{s}, \mathrm{r}\}$ and $\mathrm{j} \in\{\mathrm{r}, \mathrm{d}\}$.

We consider the scenario in which the fading coefficients are known, i.e., accurately measured by the appropriate receivers, but not fully known to, or not exploited by, the transmitters.

We model $a_{i, j}$ statistically as zero-mean, independent, circularly-symmetric complex Gaussian random variables with variances $\sigma_{i, j}^{2}$. Also, we model $z_{j}[n]$ as zero-mean mutually independent, circularlysymmetric, complex Gaussian random sequences with variance $N_{0}$.

\section{PARAMETERIZATIONS}

Two important parameters of the system are 1. SNR without fading and 2. Spectral efficiency.

We nowdefine these parameters in terms of standard parameters in the continuous-time channel. For a continuoustime channel with bandwidth $\mathrm{W} \mathrm{Hz}$ available for transmission, the discrete-time model contains Wtwodimensional symbols per second $(2 \mathrm{D} / \mathrm{s})$.

For Amplify-and-Forward transmission, the appropriate channel model is equations (2)-(4). The source terminal transmits its information $\operatorname{asx}_{\mathrm{s}}[\mathrm{n}]$, say, for $\mathrm{n}=1, \ldots, \mathrm{N} / 4$.

During this interval, the relay processesy $_{r}[n]$, and relays the information by transmitting

$$
\mathbf{x}_{\mathbf{r}}[\mathbf{n}]=\boldsymbol{\beta} \mathbf{y}_{\mathbf{r}}[\mathbf{n}-\mathbf{N} / \mathbf{4}]
$$

for $n=N / 4+1, \ldots, N / 2$.

To remain within its power constraint (with high probability), an amplifying relay must use gain

$$
\boldsymbol{\beta} \leq \sqrt{\frac{\mathbf{P}}{\left|\mathbf{a}_{\mathbf{s}, \mathbf{r}}\right| \mathbf{P}+\mathbf{N}_{\mathbf{0}}}}
$$

where we allow the amplifier gain to depend upon the fading coefficient as $r$ between the source and relay, which the relay estimates to high accuracy.

This scheme can be viewed as repetition coding from twoseparate transmitters, except that the relay transmitter amplifies its own receiver noise.

The destination can decode its received signaly $\mathbf{y}_{\mathbf{d}}[\mathbf{n}]$ for $\mathrm{n}=$ $1, \ldots, N / 2$ by first appropriately combining the signals fromthe two sub blocks using a suitably designed matched-filter (maximum-ratio combiner).

\section{RESULTS AND DISCUSSION}

We here modelled the channel with appropriate equations, and taken the outage probability equations from Table 1, and simulate the MATLAB code [2]. After the successful simulation of MATLAB code, Fig.6. below shows the results and shows the graph between BER of AF relaying. We simulated and demonstrate the Amplify and Forward cooperative protocols and results shows the robustness of this protocol to fairly general channel conditions. 


\section{International Journal of Innovative Research in Electrical, Electronics, Instrumentation and Control Engineering \\ ISO 3297:2007 Certified}

Vol. 5, Issue 2, February 2017

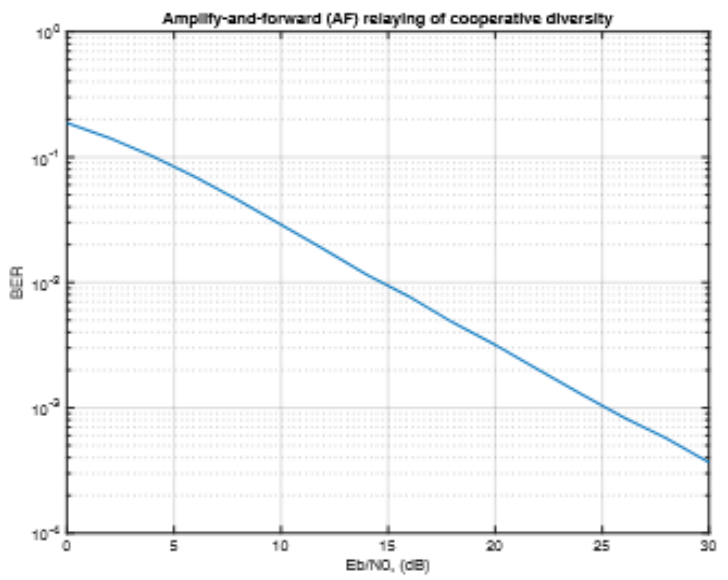

Figure 6. Bit error rate of AF relaying.

In addition to direct transmission, we examine fixed relaying protocols in which the relay amplifies what it receives and re-transmits the source message. We call these options amplify-and-forward.

These above protocols are inspired by the observation [5], [17], [26] and cooperation [5], [6], [28] schemes, respectively, but we limit the complexity of our protocols for ease of implementation. Our analysis suggests that cooperating radios may also employ threshold tests on the measured channel quality between them, to obtain adaptive protocols, called selection relaying, that choose the strategy with best performance. Relay selection and incremental relaying protocols represent new directions for relay and cooperative transmission, building upon existing ideas.

\section{REFERENCES}

[1] A. Nosratinia, T. E. Hunter, A. Hedayat, "Cooperative Communications in Wireless Networks", IEEE Communications Magazine, 2004, pp. 74-80.

[2] K. Tahir, "Coded Cooperative Communications", MSc Thesis, Blekinge Institute of Technology (BTH), Sweden, September 2007

[3] S. A. Zummo, "Performance Analysis of Coded Cooperation Diversity in Wireless Networks", Journal of Wireless Communications and Mobile Computing, John Wiley and Sons Ltd, 2005.

[4] M. Uysal, "Cooperative Communications for Improved Wireless Network Transmission”, Information Science Reference, July 2009.

[5] G. K. Karagiannidis, C. Tellambura, S. Mukherjee, A. O. Fapojuwo, "Editorial - Multi-user Cooperative Diversity for Wireless Networks", EURASIP Journal on Wireless Communications and Networking.

[6] E. C. Van Der Meulen, "Three-terminal communication channels", Advances in Applied Probability, 3:120-154, 1971.

[7] T. M. Cover and A. A. E. Gamal, "Capacity Theorems for the Relay Channel”, IEEE Trans. Info. Theory, vol. 25, no. 5, Sept. 1979, pp. 572-584.

[8] A. Meier, "Cooperative Diversity in Wireless Networks", MSc Thesis, University of Edinburgh, 2004.

[9] J. N. Laneman, G. W. Wornell and D. N. C. Tse, “An Efficient Protocol for Realizing Cooperative Diversity in Wireless Networks", Proc. IEEE ISIT, Washington, DC, June 2001, p. 294.

[10] T. E. Hunter and A. Nosratinia, "Cooperative Diversity through Coding”, Proc. IEEE ISIT, Laussane, Switzerland, July 2002, p. 220.
[11] T. E. Hunter and A. Nosratinia, "Diversity through Coded Cooperation", submitted to IEEE Trans. Wireless Commun., 2004.

[12] J. G. Proakis, "Digital Communications", McGraw Hill (4th Edition), 2001.

[13] A. Papoulis, "Probability, Random Variables, and Stochastic Processes", McGraw Hill, 1965.

[14] A. Viterbi, J. Omura, "Principles of Digital Communication and Coding”, McGraw Hill, 1979.

[15] Jakes W, "Microwave Mobile Communications", IEEE Press, 1974

[16] W. Stalling, "Wireless Communications and Networking", Pearson Education.

[17] S. Haykin, "Digital Communication Systems", John Wiley and Sons Inc, 2003.

[18] G. Kramer, M. Gastpar, "Cooperative Strategies and Capacity Theorems for Relay Networks", IEEE Trans. Information Theory, vol. 51, no .9, September 2005.

[19] M. Janani, A. Hedayat, T. E. Hunter and A. Nosratinia, "Coded cooperation in wireless communications: Space-time transmission and iterative decoding", IEEE Trans. Signal Processing, vol. 52, no. 2, pp. 362-371, Feb. 2004

[20] A. del Coso, C. Ibars, "Capacity of decode-and-forward cooperative links with full channel state information", Proc. 39th Asilomar Conference on Signals, Systems and Computers, Pacific Grove, CA, Nov. 4-6 2005.

[21] T. M. Cover and A. A. E. Gamal, "Capacity Theorems for the Relay Channel", IEEE Trans. Info. Theory", vol. 25, no. 5, Sept. 1979 , pp. $572-84$

[22] J. N. Laneman, D. Tse, "Cooperative diversity in wireless networks: efficient protocols and outage behaviour", IEEE Trans. Information Theory, vol. 50, no. 12, December 2004.

[23] J. N. Laneman and G. W. Wornell, "Distributed space-time coded protocols for exploiting cooperative diversity in wireless networks", IEEE Trans. Inform. Theory, vol. 49, pp. 2415-2525, Oct. 2003.

[24] R. U. Nabar and H. Bölcskei, "Fading relay channels: performance limits and Space-Time signal design", IEEE Journal on Selected Areas in Communications, Vol. 22, no. 6, August 2004.

[25] B. Zhao and M. Valenti, "Some New Adaptive Protocols for the Wireless Relay Channel", Proc. Allerton Conf. Commun., Control, and Comp., Monticello, IL, Oct. 2003.

[26] P. Larsson, "Selection Diversity Forwarding in a Multihop Packet Radio Network with Fading Channel and Capture", Mobile Comp. Commun. Rev., vol. 5, no. 4, Oct. 2001, pp. 47-54.

[27] S. Wei, D. L. Goeckel, M. C. Valenti, "Asynchronous cooperative diversity”, IEEE Trans. Wireless Commun., vol. 5, pp. 1547-1557, June 2006

[28] R. U. Nabar, F. W. Kneubuhler, H. Bölcskei, "Performance limits of amplify-and-forward based fading relay channels", IEEE Proc. ICASSP, vol. 4, pp. iv-565 - iv-568, May 2004.

[29] Qian Zhang, Juncheng Jia, Jin Zhang, "Cooperative relay to improve diversity in cognitive radio networks," IEEE Communications Magazine, vol. 47, issue. 2, 2009, pp. 111-117.

[30] Y. W Hong, W. J. Huang, F. H. Chiu, C. C. J. Kuo, "Cooperative Communications in Resource-Constrained Wireless Networks", IEEE Signal Processing Magazine, vol. 24, issue. 3, 2007, pp. 4547.

[31] A. Bletsas, A. Lippman, "Implementing cooperative diversity antenna arrays with commodity hardware", IEEE Communications Magazine, vol. 24, issue. 12, 2006, pp. 33-40.

[32] R. Pabst, B. H. Walke, D. C. Schultz, P. Herhold, H Yanikomeroglu, S. Mukherjee, et.al.,"Relay-based deployment concepts for wireless and mobile broadband radio", IEEE Communications Magazine, vol. 42, issue. 09, 2004, pp. 80-89.

[33] Yonghui Li, "Distributed coding for cooperative wireless networks: An overview and recent advances", IEEE Communications Magazine, vol. 47, issue. 08, 2009, pp. 71-77. 